\title{
USO DE LOS SERVICIOS DE SALUD AMBULATORIOS EN LA POBLACIÓN PERUANA
}

\author{
Pedro Ypanaqué-Luyo ${ }^{1, a}$, Mônica Martins $2, b$
}

\begin{abstract}
RESUMEN
Objetivos. Describir el perfil de necesidad y los patrones de utilización de los servicios de salud ambulatorios en la población afiliada y no afiliada a los seguros de salud en el Perú. Materiales y métodos. Estudio transversal descriptivo usando los datos de la Encuesta Nacional de Hogares 2011. Los datos se distribuyeron en cinco grupos: personas afiliadas al Seguro Social de Salud (EsSalud), Seguro Integral de Salud (SIS), Seguro de Salud de las Fuerzas Armadas y Policiales (FA-FP), los seguros privados de salud (SPS) y personas no aseguradas. Resultados. Personas aseguradas y las afiliadas al EsSalud tuvieron más probabilidades de usar la consulta ambulatoria que las personas no aseguradas y los afiliados al SIS. Los afiliados al SIS acudieron principalmente a los centros y puestos de salud $(47,1 \%)$, mientras que los afiliados a otros seguros utilizaron mayoritariamente los hospitales y clínicas. Los no asegurados usaron como primera opción las farmacias y boticas $(46,7 \%)$ con la finalidad de solucionar sus problemas de salud. En todos los seguros se realizó pago de bolsillo por la consulta, los medicamentos y los exámenes auxiliares. La percepción de "no gravedad" fue la principal razón de no búsqueda de atención en los asegurados $(40,0 \%)$ y no asegurados $(41,4 \%)$; no obstante la falta de dinero mostró una diferencia importante entre asegurados $(10,6 \%)$ y no asegurados (16,3\%). Conclusiones. El perfil de utilización de los servicios de salud ambulatoria en la población peruana expresa diferencias que se relacionan con la tenencia y el tipo de seguro.
\end{abstract}

Palabras clave: Accesibilidad a los servicios de salud; Seguro de salud; Atención ambulatoria (fuente: DeCS BIREME).

\section{UTILIZATION OF OUTPATIENT HEALTH SERVICES IN THE PERUVIAN POPULATION}

\begin{abstract}
Objectives. Describe the profile of need and patterns of use of outpatient health services in populations who are enrolled and non-enrolled in a health insurance program in Peru. Materials and methods. Descriptive cross-sectional study using data from the 2011 National Household Survey. The data were divided into five groups: persons enrolled in the Social Health Insurance (ESSALUD), Integral Health Insurance (SIS), Health Insurance of the Armed Forces and Police Force (AF-PF), Private Health Insurances (SPS) and the uninsured. Results. People insured and enrolled in ESSALUD were more likely to use outpatient services than uninsured people and those enrolled in SIS. SIS members mainly attended health centers and small health posts ( $47.1 \%$ ), while other insurance members mainly used hospitals and private hospitals. The uninsured used as their first choice pharmacies and drugstores (46.7\%) in order to solve their health problems. In all insurances, out-of-pocket payment was made for the consultation, medications and tests. The perception of "not serious" was the main reason for not seeking care in the insured (40.0\%) and uninsured (41.4\%); however the lack of money showed an important difference between insured (10.6\%) and uninsured (16.3\%). Conclusions. The outpatient health services utilization profile of the Peruvian population expresses differences that relate to enrollment in and type of insurance.
\end{abstract}

Key words: Health services accessibility; Insurance, health; Ambulatory care (source: MeSH NLM).

\section{INTRODUCCIÓN}

En el Perú, el sistema de seguros de salud está constituido por diferentes entidades públicas y privadas, tales como el Seguro Integral de Salud (SIS), el Seguro Social de Salud (EsSalud), el Fondo de Salud del Personal de la Policía Nacional (FOSPOLI), el Fondo de Salud del
Personal Militar de las Fuerzas Armadas (FOSPEMFA) y un conjunto de seguros privados de distinta naturaleza y alcance. En la última década, el Estado ha impulsado una importante política de expansión del SIS, logrando que el porcentaje de la población asegurada se incremente entre los años $2000(32 \%)$ y $2011(66 \%)^{(1-3)}$. No obstante, el $34 \%$ de la población no está asegurada, quedando en

\footnotetext{
Dirección de Servicios de Salud, Ministerio de Salud. Lima, Perú.

Escola Nacional de Saúde Pública, Fundação Oswaldo Cruz. Rio de Janeiro, Brasil.

a Magíster en Salud Pública; ${ }^{\text {b }}$ doctor en Salud Pública

El presente artículo se desarrolló sobre la base de la tesis presentada por PYL a la Maestría de Salud Pública organizada por el Instituto Nacional de Salud y Fiocruz.

Recibido: : 14-09-14 Aprobado: 13-05-15
}

Citar como: Ypanaqué-Luyo P, Martins M. Uso de los servicios de salud ambulatorios en la población peruana. Rev Peru Med Exp Salud Publica. 2015;32(3):464-70. 
este segmento los trabajadores del sector informal, los autoempleados y los desempleados, con sus familias.

Una forma de estudiar el acceso a los servicios de salud, es a través del análisis del uso de los servicios de salud, lo cual permite medir la "demanda efectiva" que se desencadena cuando las personas buscan atención por una necesidad de salud ${ }^{(5)}$. Uno de los modelos conceptuales más usados para este análisis es el propuesto por Ronald M. Andersen ${ }^{(6)}$ que establece tres principales factores determinantes del uso de los servicios de salud a nivel por parte de los individuos: i) los factores predisponentes; ii) los factores mediadores que facilitan o impiden; y iii) las necesidades de salud. Los factores predisponentes se agrupan en tres bloques: i) los factores demográficos (ej. edad, sexo, tamaño familiar); ii) la estructura social (ej. clase social, educación, ocupación), y iii) las creencias en salud. Mientras que los factores mediadores se agrupan en dos bloques: i) los vinculados a la persona y la familia (ej. ingresos familiares, cobertura del seguro), y ii) los vinculados a la comunidad (ej. ruralidad, distancia al establecimiento de salud). El modelo reconoce las necesidades de salud (percepción de enfermedad, riesgo o gravedad) como los determinantes más proximales que desencadenan el uso de los servicios de salud).

Una revisión realizada por Moreno-Serra \& Smith (7) encontró que la cobertura de salud universal ha contribuido a mejorar la salud de la población al reducir las barreras financieras del acceso a los servicios (atención preventiva, consulta y hospitalización) y por favorecer mejores resultados en la población pobre. No obstante, el autor hace la precisión que estos resultados están muy influenciados por el marco institucional y la gobernanza lograda en cada país; así como, la existencia de mecanismos de riesgo compartido (fondos de salud). Al respecto, en el Perú se han realizado estudios sobre el perfil de uso de los servicios de salud que han identificado desigualdades en el acceso y uso de estos servicios entre la población asegurada y no asegurada ${ }^{(8-10)}$.

Con la finalidad de aportar información sobre las diferencias en el acceso a los servicios que existe entre los diferentes seguros de salud en el Perú, el objetivo del presente artículo es describir y comparar el perfil de necesidad y los patrones de uso de los servicios de salud ambulatorios en la población afiliada y no afiliada a los seguros de salud en el Perú.

\section{MATERIALES Y MÉTODOS}

Estudio transversal descriptivo. Se emplearon datos de la Encuesta Nacional de Hogares (ENAHO) del año 2011, que fue realizada por el Instituto Nacional de Estadística e Informática (INEI) del Perú y que fueron obtenidos de una base de datos de uso público. La muestra de la ENAHO 2011 estuvo constituida por 26456 viviendas, seleccionadas a través de muestreo probabilístico, de áreas, estratificado y con tres etapas, con un nivel de confianza del $95 \%$ y una tasa de no-respuesta total del 8,3\% (11,12). La metodología de estimación para procesar los datos de la ENAHO involucra el uso de un peso o factor de expansión para cada registro que es multiplicado por todos los datos que conforman el registro correspondiente. El factor final para cada registro tiene dos componentes: el factor básico de expansión y los factores de ajuste por la no entrevista

El factor básico de expansión para cada hogar es determinado por el diseño de la muestra, y equivale al inverso de su probabilidad final de selección, el mismo que es el producto de las probabilidades de selección en cada etapa. El diseño de la muestra de la ENAHO involucra hasta tres etapas de muestreo donde las unidades son seleccionadas con probabilidades proporcionales al tamaño ( $p p t)$ excepto la última etapa. En la última etapa son seleccionadas un número de viviendas para cada conglomerado, teniendo en cuenta un intervalo de selección. Los factores de expansión básicos de la ENAHO son ajustados teniendo en cuenta las proyecciones de población por grupos de edad y sexo para cada mes de la encuesta y niveles de inferencia propuestos en el diseño de la muestra. Cabe mencionar que se podrán obtener estimaciones para otros niveles de desagregación, y su precisión o confiabilidad estadística dependerá, fundamentalmente, del número de casos u observaciones contenidas en la base de datos ${ }^{(11,12)}$.

La población de estudio quedo conformada con 30218555 personas, luego que se aplicara el factor de expansión calculado sobre el total de personas que respondieron estar afiliadas o no a un seguro de salud ( $n=67861)$.

El $64 \%$ de la población declaró tener un seguro de salud. Al respecto, la población del estudio se distribuyó en cinco grupos: las personas afiliadas al Seguro Social de Salud (EsSalud), Seguro Integral de Salud (SIS), Seguro de Salud de las Fuerzas Armadas y Policiales (FA-FP), los seguros privados de salud (SPS) y las personas no aseguradas. Para fines del estudio los afiliados a las entidades prestadoras de salud (EPS) fueron incluidos en el grupo de los seguros privados, por tratarse de empresas privadas que ofrecen atención de salud a sus asegurados través de establecimientos de salud privados. El presente estudio no consideró el análisis de las personas que estaban afiliadas al mismo tiempo a diferentes seguros de salud (multiafiliados). Al respecto, en la población de estudio se encontró que el 1,7\% declaró estar afiliado a dos seguros y el $0,04 \%$ declaró estar afiliado a tres seguros de salud.

Los datos se analizaron por cada grupo de estudio y de manera comparativa entre los diferentes grupos. Se 
Tabla 1. Perfil demográfico por tenencia y tipo de seguro. Perú, 2011

\begin{tabular}{|c|c|c|c|c|c|c|c|c|c|c|}
\hline \multirow{2}{*}{ Características } & \multicolumn{2}{|c|}{ Seguro Integral } & \multicolumn{2}{|c|}{ Seguro Social } & \multicolumn{2}{|c|}{ Seguro Militar-Policial } & \multicolumn{2}{|c|}{ Seguro Privado } & \multicolumn{2}{|c|}{ Sin Seguro de Salud } \\
\hline & $\mathbf{N}$ & $(\%)$ & $\mathbf{N}$ & $(\%)$ & $\mathbf{N}$ & $(\%)$ & $\mathbf{N}$ & $(\%)$ & $\mathbf{N}$ & $(\%)$ \\
\hline \multicolumn{11}{|l|}{$\overline{\text { Sexo }}$} \\
\hline Hombre & 5014925 & $(45,8)$ & 3591101 & $(49,0)$ & 359836 & $(55,7)$ & 548797 & $(52,9)$ & 5624708 & $52,2)$ \\
\hline Mujer & 5935525 & $(54,2)$ & 3740122 & $(51,0)$ & 286137 & $(44,3)$ & 489034 & $(47,1)$ & 5154958 & $47,8)$ \\
\hline Total & 10950450 & (100) & 7331223 & $(100)$ & & $(100)$ & 1037831 & $100)$ & 10779666 & (100) \\
\hline \multicolumn{11}{|l|}{ Grupo etario } \\
\hline 0 a 4 & 1267150 & $(11,6)$ & 521702 & $(7,1)$ & 23658 & $(3,7)$ & 56997 & $(5,5)$ & 582645 & $(5,4)$ \\
\hline 5 a 14 & 2992111 & $(27,3)$ & 1182014 & $(16,1)$ & 111218 & $(17,2)$ & 122561 & $(11,8)$ & 1451165 & $(13,5)$ \\
\hline 15 a 24 & 2001598 & $(18,3)$ & 768888 & $(10,5)$ & 123052 & $(19,0)$ & 273276 & $(26,3)$ & 2572537 & $(23,9)$ \\
\hline 25 a 49 & 2774624 & $(25,3)$ & 2603612 & $(35,5)$ & 1905 & $(29,5)$ & 382348 & $36,8)$ & 3772595 & $(35,0)$ \\
\hline 50 a 64 & 1071813 & $(9,8)$ & 1276325 & $(17,4)$ & 115340 & $(17,9)$ & 132395 & $12,8)$ & 1552719 & $(14,4)$ \\
\hline 65 y más. & 843155 & $(7,7)$ & 978683 & $(13,4)$ & 82162 & $(12,7)$ & 70253 & $6,8)$ & 848005 & $(7,8)$ \\
\hline Total & 10950451 & (100) & 7331224 & (100) & 645973 & $(100)$ & 1037830 & 100) & 10779666 & (100) \\
\hline
\end{tabular}

Nota: $\mathrm{N}^{\circ}$ valores totales $=30266323 . \mathrm{N}^{\circ}{ }^{\circ}$ valores perdidos $=0$.

analizaron las variables que están relacionadas con el consumo de los servicios de salud, tales como: las características demográficas, geográficas y educativas; las necesidades de salud medidas a través de la autoevaluación del estado de salud y el uso de la consulta ambulatoria en las últimas 4 semanas. Las variables "lugar de residencia", "ingreso", "tipo de ocupación", "tipo de contrato" y "tipo de empleador de la ocupación principal", no fueron analizadas al no ser posible la fusión de la base de datos del módulo salud con los módulos de vivienda y empleo de la ENAHO 2011, debido a que la base de datos de los otros cuestionarios no incluye a todos los miembros del hogar.

Se empleó la estadística descriptiva y se estimaron odds ratios (OR) para algunas variables. Para el análisis estadístico se utilizó el software SPSS Statistics 22.

El estudio fue presentado y aprobado por el Comité Institucional de Ética del Instituto Nacional de Salud (Registro 9183-2013).

\section{RESULTADOS}

\section{CARACTERISTICAS DEMOGRÁFICAS, GEOGRÁFICAS Y EDUCATIVAS}

EI SIS presentó una importante proporción de personas entre 0 y 14 años $(38,9 \%)$, procedentes de la sierra $(49,8 \%)$ y la selva $(20,3 \%)$; del sexo femenino $(54,2 \%)$; con educación primaria $(44,4 \%)$ y sin nivel educativo $(12,7 \%)$. En los otros seguros predominaron las personas adultas o adultas mayores, procedentes de la costa y con educación secundaria o superior. En el Seguro Social de Salud $(51,0 \%)$ predominaron las mujeres, mientras que en el Seguro Militar-Policial $(55,7 \%)$ y el seguro privado $(52,9 \%)$ predominaron los varones. Al respecto, estos hallazgos se relacionan con el tipo de población objetivo al cual está dirigido cada seguro de salud. En la población no asegurada predominó el grupo de 15 a 49 años, procedentes de la costa $(64,5 \%)$, con educación secundaria $(42,1 \%)$ y con predominio del sexo masculino $(52,2 \%)$ (Tabla 1 y 2$)$.

Tabla 2. Aspectos geográficos y educativos, por tenencia y tipo de seguro. Perú, 2011

\begin{tabular}{|c|c|c|c|c|c|c|c|c|c|c|}
\hline \multirow[t]{2}{*}{ Características } & \multicolumn{2}{|c|}{$\begin{array}{l}\text { Seguro } \\
\text { Integral }\end{array}$} & \multicolumn{2}{|c|}{$\begin{array}{l}\text { Seguro } \\
\text { Social }\end{array}$} & \multicolumn{2}{|c|}{$\begin{array}{c}\text { Seguro } \\
\text { Militar-Policial }\end{array}$} & \multicolumn{2}{|c|}{$\begin{array}{l}\text { Seguro } \\
\text { Privado }\end{array}$} & \multicolumn{2}{|c|}{$\begin{array}{c}\text { Sin Seguro de } \\
\text { Salud }\end{array}$} \\
\hline & $\mathbf{N}$ & $(\%)$ & $\mathbf{N}$ & $(\%)$ & $\mathbf{N}$ & $(\%)$ & $\mathbf{N}$ & $(\%)$ & $\mathbf{N}$ & $(\%)$ \\
\hline \multicolumn{11}{|l|}{ Región natural $^{1}$} \\
\hline Resto costa & 2057363 & $(18,8)$ & 1933598 & $(26,4)$ & 115284 & $(17,8)$ & 98330 & $(9,5)$ & 2874669 & $(26,6)$ \\
\hline Sierra & 5453162 & $(49,8)$ & 1606260 & $(21,9)$ & 82447 & $(12,8)$ & 125365 & $(12,1)$ & 2681896 & $(24,9)$ \\
\hline Selva & 2224827 & $(20,3)$ & 549153 & $(7,5)$ & 38951 & $(6,0)$ & 48769 & $(4,7)$ & 1138783 & $(10,6)$ \\
\hline Lima Metropolitana & 1215097 & $(11,1)$ & 3242212 & $(44,2)$ & 409292 & $(63,4)$ & 765368 & $(73,7)$ & 4084318 & $(37,9)$ \\
\hline Total & 10950449 & $(100)$ & 7331223 & $(100)$ & 645974 & $(100)$ & 1037832 & $(100)$ & 10779666 & $(100)$ \\
\hline \multicolumn{11}{|l|}{ Nivel educativo ${ }^{2}$} \\
\hline Sin nivel & 1297856 & $(12,7)$ & 265141 & $(3,8)$ & 13747 & $(2,2)$ & 12369 & $(1,2)$ & 652417 & $(6,3)$ \\
\hline Inicial & 570479 & $(5,6)$ & 309318 & $(4,4)$ & 16074 & $(2,5)$ & 32604 & $(3,2)$ & 301478 & $(2,9)$ \\
\hline Primaria & 4531258 & $(44,4)$ & 1528628 & $(21,7)$ & 110911 & $(17,5)$ & 98963 & $(9,9)$ & 2865046 & $(27,5)$ \\
\hline Secundaria & 3213812 & $(31,5)$ & 2278331 & $(32,4)$ & 249636 & $(39,4)$ & 222610 & $(22,2)$ & 4384717 & $(42,1)$ \\
\hline Superior no universitaria & 394590 & $(3,9)$ & 1222604 & $(17,4)$ & 106958 & $(16,9)$ & 154664 & $(15,4)$ & 1122151 & $(10,8)$ \\
\hline Superior universitaria & 194803 & $(1,9)$ & 1424584 & $(20,3)$ & 136494 & $(21,5)$ & 482466 & $(48,1)$ & 1096693 & $(10,5)$ \\
\hline Total & 10202798 & $(100)$ & 7028606 & $(100)$ & 633820 & $(100)$ & 1003676 & $(100)$ & 10422502 & $(100)$ \\
\hline
\end{tabular}

(1) $\mathrm{N} .^{\circ}$ valores totales $=30266323 . \mathrm{N} .^{\circ}$ valores perdidos $=0$.

(2) $\mathrm{N} .^{\circ}$ valores totales $=30266323 . \mathrm{N} .^{\circ}$ valores válidos= $28799215 .{ }^{\circ} .^{\circ}$ valores perdidos=1467 108. 
Tabla 3. Necesidades de salud y uso de la consulta. Perú, 2011

\begin{tabular}{|c|c|c|c|c|c|c|}
\hline \multirow[t]{2}{*}{ Tenencia y tipo de seguro } & \multicolumn{2}{|c|}{$\begin{array}{c}\text { Problemas de salud agudos }<4 \\
\text { semanas }^{1}\end{array}$} & \multicolumn{2}{|c|}{$\begin{array}{l}\text { Problemas de salud } \\
\text { crónicos }^{1}\end{array}$} & \multicolumn{2}{|c|}{$\begin{array}{c}\text { Utilización de la consulta }<4 \\
\text { semanas }\end{array}$} \\
\hline & $\mathbf{N}$ & $(\%)$ & $\mathbf{N}$ & $\%$ & $\mathbf{N}$ & $(\%)$ \\
\hline Con seguro de salud & 11943976 & $(61,4)$ & 7115140 & $(36,6)$ & 4534541 & $(34,8)$ \\
\hline Seguro Integral de Salud & 6830841 & $(62,4)$ & 3143141 & $(28,7)$ & 2354700 & $(31,0)$ \\
\hline Seguro Social de Salud & 4498420 & $(61,4)$ & 3481434 & $(47,5)$ & 1968905 & $(41,3)$ \\
\hline Seguro Militar y Policial & 349907 & $(54,2)$ & 301902 & $(46,7)$ & 140353 & $(36,9)$ \\
\hline Seguro Privado de Salud & 568366 & $(54,8)$ & 464672 & $(44,8)$ & 233345 & $(39,0)$ \\
\hline Sin seguro de salud & 6327248 & $(58,7)$ & 3816217 & $(35,4)$ & 1292030 & $(19,1)$ \\
\hline Total & 18271224 & $(60,5)$ & 10931357 & $(36,2)$ & 5826571 & $(29,5)$ \\
\hline
\end{tabular}

1: N. ${ }^{\circ}$ valores válidos $=30218555 . \mathrm{N} .^{\circ}$ valores perdidos $=47768$

2: $\mathrm{N} .^{\circ}$ valores válidos $=19782411 . \mathrm{N} .^{\circ}$ valores perdidos=10 483911.

\section{NECESIDADES DE SALUD}

Reportaron más problemas de salud agudos los afiliados al SIS $(62,4 \%)$ frente a los afiliados al seguro social $(61,4 \%)$, el seguro privado $(54,8 \%)$ y el seguro militar y policial $(54,2 \%)$. Asimismo, reportaron más problemas de salud crónicos los afiliados al Seguro Social de Salud $(47,5 \%)$ frente al seguro militar y policial $(46,7 \%)$, el seguro privado $(44,8 \%)$ y el SIS $(28,7 \%)$. De otro lado, reportaron más problemas de salud agudos la población asegurada $(61,4 \%)$ que la población no asegurada $(58,7 \%)$; asimismo, los asegurados reportaron más problemas de salud crónicos $(36,6 \%)$ que los no asegurados $(35,4 \%)$ (Tabla 3$)$.

\section{USO DE LA CONSULTA AMBULATORIA}

Al analizar el uso de la consulta ambulatoria en las últimas 4 semanas, se encontró mayor uso en los afiliados al Seguro Social de Salud $(41,3 \%)$, el seguro privado $(39,0 \%)$ y el Seguro Militar y Policial $(36,9 \%)$, y un menor uso en los afiliados al SIS $(31,0 \%)$ y no asegurados $(19,1 \%)$ (Tabla 3 ). Al respecto, tuvieron mayor probabilidad de usar la consulta ambulatoria las personas con un seguro de salud (OR=2,26, IC $95 \%$ : $2,26-2,27)$ comparativamente con los no asegurados. También se aprecia que tuvieron más probabilidad de utilizar la consulta ambulatoria los afiliados al Seguro Social de Salud (OR=1,56; IC 95\%: 1,55-1,56), las mujeres (OR=1,21: IC 95\%: 1,20-1,21), los menores de 4 años (OR=2,99; IC 95\%: 2,97-3,00), los mayores de 65 años (OR=2,24; IC 95\%: 2,22-2,24), los residentes de Lima Metropolitana (OR=1,37; IC 95\%: 1,36-1,37) y las personas con problemas de salud crónicos $(\mathrm{OR}=1,68$; IC 95\%: 1,68-1,68) (Tabla 4).

\section{LUGAR DE LA CONSULTA}

Los afiliados al SIS (42,6\%) usaron principalmente los centros y puestos de salud de la red pública de atención primaria; mientras que los afiliados al Seguro
Tabla 4. Estimación de Odds Ratio en la consulta ambulatoria Perú, 2011

\begin{tabular}{|c|c|c|}
\hline Variables & $\begin{array}{l}\text { Uso de la consulta } \\
\text { ambulatoria \% }\end{array}$ & OR (IC $95 \%)$ \\
\hline \multicolumn{3}{|l|}{ Tenencia de seguro } \\
\hline Sí tiene seguro & 77,8 & $2,26(2,26-2,27)$ \\
\hline No tiene seguro & 22,2 & 1,00 \\
\hline \multicolumn{3}{|l|}{ Tipo de seguro } \\
\hline Seguro social & 45,5 & $1,56(1,55-1,56)$ \\
\hline Seguro integral & 54,5 & 1,00 \\
\hline Seguro social & 89,4 & $1,10(1,09-1,10)$ \\
\hline Seguro privado & 10,6 & 1,00 \\
\hline \multicolumn{3}{|l|}{ Sexo } \\
\hline Mujer & 60,7 & $1,21(1,20-1,21)$ \\
\hline Hombre & 39,3 & 1,00 \\
\hline \multicolumn{3}{|l|}{ Educación } \\
\hline Superior universitaria & 51,5 & $1,04(1,03-1,04)$ \\
\hline Sin educación & 48,5 & 1,00 \\
\hline \multicolumn{3}{|l|}{ Región } \\
\hline Lima Metropolitana & 43,5 & $1,36(1,36-1,37)$ \\
\hline Sierra y Selva & 56,5 & 1,00 \\
\hline \multicolumn{3}{|l|}{ Grupo etario } \\
\hline 0 a 4 & 53,8 & $2,99(2,97-3,00)$ \\
\hline 15 a 24 & 46,2 & 1,00 \\
\hline 65 y más. & 53,3 & $2,23(2,22-2,24)$ \\
\hline 15 a 24 & 46,7 & 1,00 \\
\hline \multicolumn{3}{|c|}{ Problema de salud agudo } \\
\hline Sí & 100,0 & - \\
\hline No & 0,0 & - \\
\hline \multicolumn{3}{|c|}{ Problema de salud crónico } \\
\hline Sí & 51,4 & $1,68(1,68-1,68)$ \\
\hline No & 48,6 & 1,00 \\
\hline
\end{tabular}

Social $(34,2 \%)$, el Seguro Militar y Policial $(33,5 \%)$ y el seguro privado $(39,6 \%)$ usaron con más frecuencia los hospitales y clínicas de sus redes asistenciales. Las personas no aseguradas usaron mayoritariamente las farmacias y boticas $(46,7 \%)$ y en segundo lugar los establecimientos de salud de atención primaria $(24 \%)$ (Tabla 5). 
Tabla 5. Lugar de la consulta ambulatoria. Perú, 2011

\begin{tabular}{|c|c|c|c|c|c|c|c|c|c|c|c|c|}
\hline \multirow{2}{*}{ Lugar de consulta } & \multicolumn{2}{|c|}{$\begin{array}{l}\text { Seguro } \\
\text { integral }\end{array}$} & \multicolumn{2}{|c|}{$\begin{array}{l}\text { Seguro } \\
\text { social }\end{array}$} & \multicolumn{2}{|c|}{$\begin{array}{c}\text { Seguro } \\
\text { militar-policial }\end{array}$} & \multicolumn{2}{|c|}{$\begin{array}{l}\text { Seguro } \\
\text { privado }\end{array}$} & \multicolumn{2}{|c|}{$\begin{array}{l}\text { Sin seguro } \\
\text { de salud }\end{array}$} & \multicolumn{2}{|c|}{ Total } \\
\hline & $\mathbf{N}$ & (\%) & $\mathbf{N}^{\circ}$ & (\%) & $\mathbf{N}^{\circ}$ & $\%)$ & $\mathbf{N}^{\circ}$ & $(\%)$ & $\mathbf{N}$ & $(\%)$ & $\mathbf{N}$ & $(\%)$ \\
\hline Hospital MINSA & 283568 & $(6,9)$ & 95439 & $(3,3)$ & 10953 & $4,7)$ & 12730 & $(3,7)$ & 234146 & $(7,0)$ & 629075 & $(5,9)$ \\
\hline $\begin{array}{l}\text { Centro o Puesto de } \\
\text { Salud MINSA * }\end{array}$ & 2349424 & $(42,6)$ & 153785 & $(5,3)$ & 17745 & $7,7)$ & 5352 & $(1,5)$ & 438227 & $(13,1)$ & 425 & $(21,9)$ \\
\hline Hospital EsSalud & 6770 & $(0,2)$ & 990087 & $(34,2)$ & 4866 & $2,1)$ & 26059 & $(7,5)$ & 3236 & $(0,1)$ & 997956 & $(9,3)$ \\
\hline Posta o policlínico EsSalud & 3789 & $(0,1)$ & 286918 & $(9,9)$ & 4910 & $2,1)$ & 3109 & $(0,9)$ & 6338 & $(0,2)$ & 297671 & $(2,8)$ \\
\hline Hospital militar-policial & 1014 & $(0,0)$ & 10586 & $(0,4)$ & 77377 & $33,5)$ & 6731 & $(1,9)$ & 0 & $(0,0)$ & 77377 & $(0,7)$ \\
\hline Clínica particular & 95838 & $(2,3)$ & 232476 & $(8,0)$ & 18038 & $7,8)$ & 137806 & $(39,6)$ & 256404 & $(7,6)$ & 667073 & $(6,2)$ \\
\hline $\begin{array}{l}\text { Consultorio médico } \\
\text { particular }\end{array}$ & 179685 & $(4,4)$ & 280609 & $(9,7)$ & 16195 & $7,0)$ & 53899 & $(15,5)$ & 359724 & $(10,7)$ & 861992 & $(8,0)$ \\
\hline Farmacia o botica & 943065 & $(23,0)$ & 626125 & $(21,6)$ & 56614 & $24,5)$ & 66541 & $(19,1)$ & 1565608 & $(46,7)$ & 3235761 & $(30,2)$ \\
\hline En el hogar & 9921 & $(0,2)$ & 12034 & $(0,4)$ & 1304 & $0,6)$ & 9747 & $(2,8)$ & 6973 & $(0,2)$ & 36702 & $(0,3)$ \\
\hline Sitio de medicina tradicional & 41345 & $(1,0)$ & 9178 & $(0,3)$ & 605 & $0,3)$ & 1482 & $(0,4)$ & 38547 & $(1,1)$ & 91157 & $(0,9)$ \\
\hline
\end{tabular}

$\mathrm{N} .^{\circ}$ valores totales $=30266323$. N. $^{\circ}$ valores válidos $=19782411 . \mathrm{N} .^{\circ}$ valores perdidos=10 483912.

* Los centros o puestos del MINSA incluyen los administrados por Comités Locales de Administración en Salud (CLAS).

\section{RAZONES DE NO BÚSQUEDA DE ATENCIÓN}

La percepción de no gravedad, que es un aspecto de las necesidades de salud, fue la principal razón de no búsqueda en los afiliados al SIS $(35,4 \%)$, el Seguro Social de Salud $(46,0 \%)$, el Seguro Militar y Policial $(53,0 \%$ ) y el seguro privado de salud $(52,9 \%)$, incluyendo la población no asegurada $(41,4 \%)$. Al agruparse las respuestas, se encontró que en los asegurados y no asegurados las razones más influyentes fueron la percepción de no gravedad, los problemas asociados a la oferta (está lejos, demoran mucho en atender, no confía en médicos, maltrato del personal y falta de tiempo) y el autotratamiento (se autorecetó y prefiere curarse con remedios caseros), mientras que la falta de dinero fue una razón secundaria para la no búsqueda de atención (Figura 1).

\section{FORMA DE PAGO POR LA CONSULTA}

En todos los seguros de salud se encontró que un segmento de afiliados realizó un pago de bolsillo total o parcial por la

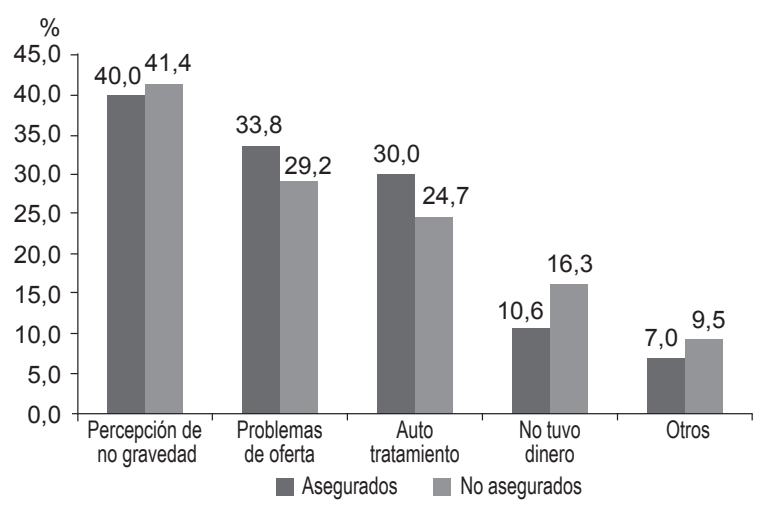

Figura 1. Razones de no búsqueda de atención. Perú, 2011 consulta, por los medicamentos y los exámenes auxiliares. La proporción de afiliados que realizaron estos pagos por toda la consulta fue mayor en el Seguro Privado $(36,6 \%)$ y el Seguro Militar y Policial $(32,9 \%)$ y algo menor en el Seguro Social $(26,9 \%)$ y el SIS $(19,9 \%)$, mientras que en la población no asegurada esta proporción fue bastante elevada $(84,9 \%)$. También se encontró que el porcentaje de afiliados al SIS $(64,0 \%)$ que realizaron pago de bolsillo por todos los exámenes de rayos $\mathrm{X}$ fue mucho mayor que en el Seguro Militar y Policial $(44,2 \%)$, el seguro privado $(26,1 \%)$, y el Seguro Social $(25,4 \%)$.

\section{DISCUSIÓN}

La literatura reporta que existe un mayor uso de los servicios de salud en los niños, los ancianos, las mujeres (principalmente en la edad fértil debido a motivos obstétricos o ginecológicos) y en las personas que disponen de un seguro de salud ${ }^{(12,13)}$. También se reporta que el empleo y la posición en el mercado de trabajo son factores que influyen en el uso de los servicios ${ }^{(14)}$ y que las personas con empleos informales y las personas sin empleo presentan un peor estado de salud autorreferido, así como una menor búsqueda y uso de los servicios de salud ${ }^{(15)}$.

Las clases sociales más bajas, al tener una mayor morbilidad y mortalidad, pueden presentar una mayor necesidad de consultar, no obstante, esta situación puede cambiar según el país, pues refleja la equidad en el uso del sistema y de los servicios de salud. Por ejemplo, Schoen et al. ${ }^{(16)}$ encontraron en Estados Unidos, Australia y Nueva Zelanda que el uso de los servicios era 2 a 3 veces mayor en las personas con mayores ingresos, mientras que en Canadá e Inglaterra el uso fue similar entre los grupos de mayor y menor ingreso. 
El presente estudio encontró que existe una mayor probabilidad de usar la consulta en las personas aseguradas, las mujeres, las personas con mayor nivel educativo, las personas en los extremos de la vida y los residentes de Lima Metropolitana y las personas con problemas de salud crónicos. Al respecto, estos hallazgos son muy similares a los reportados por otros estudios realizados en el Perú ${ }^{(3,8-10,17)}$. De esta manera, el estudio constató que los factores predisponentes (sexo, edad, educación), los factores mediadores (tenencia y tipo de seguro, urbanidad) y las necesidades de salud (percepción de enfermedad) tienen un importante efecto sobre el uso de los servicios de salud, conforme lo establece el modelo de Andersen.

El estudio encuentra que los afiliados al Seguro Social de Salud tuvieron más oportunidad de usar la consulta en comparación a los otros asegurados. De otro lado, los afiliados al SIS usaron principalmente los centros y puestos de salud de la red pública de atención primaria, mientras que los otros asegurados prefirieron los hospitales y las clínicas. Este resultado difiere a lo encontrados por Cabezas ${ }^{(18)}$.

En todos los seguros de salud se usó la farmacia o botica como un servicio sustitutorio de los establecimientos de salud. Mientras que en la población no asegurada se usó con más frecuencia la farmacia o botica, mientras que la búsqueda de un establecimiento quedo como una segunda opción. Esta situación también ha sido reportada por otros autores ${ }^{(9,19,20)}$.

Se encontró que la falta de dinero no fue la principal razón de no búsqueda de atención entre las personas aseguradas y no aseguradas; lográndose identificar otras razones más influyentes tales como: la percepción de no gravedad, los problemas relacionados con la inadecuada oferta de servicios y el autotratamiento. Que la falta de dinero sea reportada como una razón menor de no búsqueda de atención está relacionada con la expansión del SIS que ha permitido que la población pobre tenga la posibilidad de acudir a los establecimientos de salud ${ }^{(21)}$. Al respecto, la cobertura del SIS se ha incrementado del 18\% (2008) al 38\% (2011) $^{(1,2)}$; lográndose en el 2011 que el 64,3\% de "pobres", el 79,7\% de "pobres extremos" y el 59,8\% de "pobres no extremos" estuvieran afiliados al SIS (22).

Se constató que un alto porcentaje de afiliados en todos los seguros realizaron gasto de bolsillo total o parcial para cubrir los costos de la consulta, los medicamentos y los exámenes auxiliares. Este gasto de bolsillo representa el copago que las aseguradoras exigen a sus afiliados.
El estudio tuvo como limitantes su diseño descriptivo, su circunscripción a la atención ambulatoria y el uso de información secundaria de la Encuesta Nacional de Hogares (ENAHO 2011). Asimismo, se tuvieron limitaciones para obtener algunas variables por no encontrarse en la base de datos del cuestionario de salud. De otro lado, el uso de información basada en la autopercepción de los entrevistados podría introducir sesgos inherentes a la encuesta, que no son particulares del estudio. Otra limitación del estudio ha sido el uso de odds ratio para explorar las diferencias que existen entre los grupos de estudio frente a ciertas variables; no obstante, debe señalarse que existen otros métodos más específicos y apropiados para medir las desigualdades en salud. Sin embargo, a pesar de estas limitaciones, los resultados Ilaman la atención por la necesidad de plantear estudios que a través de metodologías apropiadas puedan monitorear las desigualdades e inequidades en el uso de los servicios de salud, en especial de las personas pobres afiliadas a un seguro público y de la población no asegurada. El monitoreo, ciertamente, ayudaría a implantar y evaluar políticas de salud dirigidas para minimizar les desigualdades en el uso de servicios de salud.

En conclusión, el estudio encontró que consumen más servicios de salud ambulatorios las personas que tienen un seguro de salud, con un mayor nivel educativo, las mujeres, las personas en los extremos de la vida, los enfermos crónicos y las personas que residen en Lima Metropolitana. El estudio también encontró que los afiliados al Seguro Social de Salud consumen más servicios ambulatorios que los afiliados a otros seguros de salud; que los afiliados al Seguro Integral de Salud consumen principalmente los servicios ambulatorios del primer nivel de atención, mientras que los afiliados a otros seguros de salud consumen principalmente los servicios ambulatorios de hospitales y clínicas, y que la población no asegurada consume principalmente los servicios de farmacias y boticas.

Contribuciones de autoría: PYL ha participado en la concepción y diseño del artículo, la recolección de datos, el análisis e interpretación de datos, la redacción y aprobación de la versión final. MM ha participado en el análisis e interpretación de datos, la redacción y aprobación de la versión final del artículo. Además brindó asesoría técnica en su condición de asesor de tesis.

Fuentes de financiamiento: autofinanciado.

Conflictos de interés: los autores declaran no tener conflictos de interés. 


\section{REFERENCIAS BIBLIOGRÁFICAS}

1. Alcalde-Rabanal JE, Lazo-González O, Nigenda G. Sistema de salud de Perú. Salud Publica Mex. 2011;53(supl 2): S243-54.

2. Perú, Instituto Nacional de Estadística e Informática. Condiciones de vida en el Perú. Julio-Agosto-Septiembre 2011. Lima: INEI; 2011.

3. Perú, Instituto Nacional de Estadística e Informática. Patrones de utilización de los servicios de salud. Lima: INEI; 2011.

4. Frenk J. El concepto y la medición de la accesibilidad. Salud Publica Mex. 1985;27(5):438-56.

5. Andersen R. 'Behavioral model of families' use of health services. En: Research Series $N^{\circ} 25$. Chicago: Center for Health Administration Studies. Chicago: University of Chicago; 1968.

6. Moreno-Serra R, Smith PC. Does progress towards universal health coverage improve population health? Lancet. 2012;380(9845):917-23. doi: 10.1016/S0140-6736(12)61039-3.

7. Perú, Instituto Nacional de Estadística e Informática [Internet]. Determinantes del acceso a los servicios de salud en el Perú. Lima: Instituto Nacional de Estadística e Informática; 2000 [citado el 16 de septiembre de 2012]. Disponible en: http://www.inei.gob.pe/biblioineipub/ bancopub/est/lib0387/indice.htm

8. Perú, Ministerio de Salud. Análisis y tendencias en la utilización de servicios de salud: Perú 1985-2002. Lima: Ministerio de Salud; 2003.

9. Petrera M, Valdivia M, Jimenez E, Almeida G. Equity in health and health care in Peru, 2004 - 2008. Rev Panam Salud Publica. 2013;33(2):131-6.
10. Perú, Instituto Nacional de Estadística e Informática. Perú - Encuesta Nacional de Hogares sobre condiciones de vida y pobreza. Lima: Instituto Nacional de Estadística e Informática; 2011.

11. Perú, Instituto Nacional de Estadística e Informática. Encuesta Nacional de Hogares 2011. Calidad de la Encuesta. Lima: Instituto Nacional de Estadística e Informática; 2012.

12. Mendoza-Sassi R, Béria JU. Utilización de los servicios de salud: una revisión sistemática sobre los factores relacionados. Cad Saude Publica. 2001;17(4):819-32.

13. Lima-Costa MF, Turci MA, Macinko J. Estrategia Salud de la Familia en comparación con otras fuentes de atención: indicadores de uso y calidad de los servicios de salud en Belo Horizonte, Minas Gerais, Brasil. Cad Saude Publica. 2013;29(7):1370-80.

14. Travassos C, Viacava F, Pinheiro R, Brito A. [Utilization of health care services in Brazil: gender, family characteristics, and social status]. Rev Panam Salud Publica. 2002;11(56):365-73.[Artículo en portugués]

15. Miquilin I de OC, Marín-León L, Monteiro MI, Filho HR. Desigualdades no acesso e uso dos serviços de saúde entre trabalhadores informais e desempregados: análise da PNAD 2008, Brasil. Cad Saude Publica. 2013;29(7):1392-406.

16. Schoen C, Davis K, DesRoches C, Donelan K, Blendon R. Health insurance markets and income inequality: findings from an international health policy survey. Health Policy. 2000;51(2):67-85.
17. Perú, Ministerio de Salud. Análisis y tendencias en la utilización de servicios de salud: Perú 1985-2002. Lima: MINSA; 2003.

18. Cabezas M. Equidad en la utilización de servicios de salud en el sistema general de Seguridad Social en Salud en Colombia. Tesis para obtener el grado de Maestro. Escuela Nacional de Salud Pública Sergio Arouca. Rio de Janeiro, Brasil. 2011.

19. Cortez RA. La equidad en el acceso y el financiamiento de los servicios de salud. En: Cortez, RA, ed. Salud, equidad y pobreza. Teoría y nuevas evidencias. Lima: Centro de Investigación de la Universidad del Pacifico; 2002. p. 203-47.

20. Perú, Ministerio de Salud. Análisis de la demanda y el acceso a los servicios de salud en el Perú. Lima: Ministerio de Salud; 2012.

21. Superintendencia Nacional de Aseguramiento en Salud. Anuario Estadístico 2012. Lima: Superintendencia Nacional de Aseguramiento en Salud; 2013.

22. Perú, Instituto Nacional de Estadística e Informática. Informe Técnico. Evolución de la Pobreza 2007-2011. Lima: Instituto Nacional de Estadística e Informática; 2012.

Correspondencia: Pedro Ypanaqué-Luyo. Dirección: Av. Del Aire 1025, M1, 301. San Luis, Lima, Perú.

Teléfono: (+511) 990173957

Correo electrónico:pedro.ypanaque@pucp.pe 